\title{
Gastric emptying in Type II (non-insulin-dependent) diabetes mellitus before and after therapy readjustment: no influence of actual blood glucose concentration
}

\author{
A.Holzäpfel ${ }^{1}$, A.Festa ${ }^{2}$, G.Stacher-Janotta ${ }^{1}$, H. Bergmann ${ }^{3}$, N. Shnawa ${ }^{2}$, W. Brannath ${ }^{4}$, G. Schernthaner ${ }^{2}$, \\ G.Stacher ${ }^{1}$ \\ ${ }^{1}$ Psychophysiology Unit at the Department of Surgery, University of Vienna, Austria \\ ${ }^{2}$ Department of Medicine I, Rudolf Foundation Hospital, University of Vienna, Austria \\ ${ }^{3}$ Ludwig Boltzmann Institute of Nuclear Medicine, Vienna, Austria \\ ${ }^{4}$ Institute of Medical Statistics, University of Vienna, Austria
}

\section{Abstract}

Aims/hypothesis. Hyperglycaemia that is induced short-term slows gastric emptying in healthy subjects and patients with diabetes mellitus. Little information is available on the impact of longer-lasting, naturally occurring blood glucose increases and their reduction to euglycaemic values. We studied the relation between gastric emptying and pre-prandial and postprandial blood glucose concentrations in patients with Type II (non-insulin-dependent) diabetes mellitus and secondary failure to respond to oral hypoglycaemic treatment (a) before readjusting hypoglycaemic therapy and (b) 1 week thereafter.

Methods. We studied 9 female and 1 male patient (age 60-78 years, BMI $21.9-32.5 \mathrm{~kg} / \mathrm{m}^{2}$, diabetes duration 3-33 years, $\mathrm{HbA}_{1 \mathrm{c}} 8.8-13.2 \%$ ). Gastric emptying of a radiolabelled semisolid $1168 \mathrm{~kJ}$ meal was recorded scintigraphically.
Results. Blood glucose concentration pre-prandial and postprandial was considerably lower subsequent to than before therapy readjustment in all patients (fasting, $7.9 \mathrm{mmol} / \mathrm{l} \pm 1.5 \mathrm{SD}$ vs $11.7 \pm 1.7 \mathrm{mmol} / \mathrm{l}$; $60 \mathrm{~min}$ postprandial, $11.7 \pm 2.0$ vs $15.4 \pm 2.2 \mathrm{mmol} / \mathrm{l}$ ). By contrast, gastric emptying was unchanged (residual radioactivity in stomach $50 \mathrm{~min}$ postprandial $65.7 \pm 14.1 \%$ vs $66.5 \pm 12.9 \%$ ). There was no relation between emptying and either fasting blood glucose concentration or its postprandial increase.

Conclusion/interpretation. The data do not support a major impact of actual, longer-lasting, naturally occurring blood glucose concentrations upon the rate of gastric emptying in patients with Type II diabetes. [Diabetologia (1999) 42: 1410-1412]

Keywords Non-insulin-dependent diabetes mellitus, gastric emptying, blood glucose concentration, hyperglycaemia, hypoglycaemic therapy.
Previous investigations suggested the actual blood glucose concentration to be a determinant of the rate of gastric emptying. In healthy subjects, experimentally induced hyperglycaemia slowed emptying [1-3] and in patients with Type I (insulin-dependent) diabetes mellitus, emptying was slower during induced hyperglycaemia than in euglycaemia $[3,4]$. In cross-sectional investigations in diabetic patients, these relations between naturally occurring, longer-

Received: 28 April 1999 and in revised form: 23 July 1999

Corresponding author: G. Stacher MD, Psychophysiologisches Laboratorium, Waehringer Guertel 18-20, A-1090 Wien, Austria

Abbreviations: RRA, Residual radioactivity. lasting increases in blood glucose concentrations and gastric emptying were less clear or even indiscernible [5-9]. To explain the impact of actual, longer-lasting increases in blood glucose concentrations and of their reduction on gastric emptying, we studied gastric emptying in patients with Type II (non-insulin-dependent) diabetes mellitus and secondary failure to respond to oral hypoglycaemic therapy firstly before treatment readjustment and secondly 1 week thereafter, when glycaemia was greatly decreased. 


\section{Subjects and methods}

Patients. We studied nine women and one man with Type II diabetes and secondary failure to respond to oral hypoglycaemic therapy [age 60-78 (median 71) years; duration of Type II diabetes 3-33 (median 15) years; BMI 21.9-32.5 (median 27.7) $\mathrm{kg} / \mathrm{m}^{2} ; \mathrm{HbA}_{1 \mathrm{c}} 8.8-13.2$ (median 10.7) \%, normal range 4-6\%] before and after therapy readjustment. None had signs indicative of peripheral neuropathy, had undergone operations or was taking drugs that potentially interfere with gastrointestinal motility. After admission to hospital, their therapy was readjusted by either switching to, or combining with, another hypoglycaemic agent or switching to insulin. The study was conducted according to the Declaration of Helsinki as revised in 1996.

Procedure. Patients underwent assessments of the gastric emptying of a semisolid standard meal immediately before therapy readjustment and 1 week thereafter. Capillary blood glucose was recorded just before meal ingestion and at 15-min intervals up to 120 min thereafter. Venous blood for the determination of insulin concentration by radioimmunoassay was drawn before meal ingestion. All investigations were done in the morning with patients who had finished their last meal no later than 2000 hours on the preceding day; no hypoglycaemic agents were taken in the preceding $12 \mathrm{~h}$.

Assessment of gastric emptying. The emptying of a semisolid 1168 kilo-Joule meal (250 ml milk, $15 \mathrm{~g}$ sugar, $14 \mathrm{~g}$ maize starch and cinnamon) mixed with $72 \mathrm{MBq}$ [ $\left.{ }^{99 \mathrm{~m}} \mathrm{Tc}\right]$ phytate was recorded scintigraphically [8]. Recording began at the end of meal ingestion and lasted $50 \mathrm{~min}$. Serial images over $1 \mathrm{~min}$ each were acquired in frame mode. Counts in the region of interest including the stomach were corrected for the variation in tissue attenuation caused by the changing position of the meal within the stomach, background activity, the use of a single-headed gamma camera and radionuclide decay. The counts at each time-point were related to the counts at the start of data acquisition considered as $100 \%$ and expressed as the percentage of radioactivity remaining in the stomach, i. e. residual radioactivity (RRA). The areas under the activity-time curves (AUC) were calculated as overall measures of emptying. The criterion for delayed emptying was an AUC greater than 4526 units, i. e. more than the mean AUC + 1.5 SD of 67 healthy, symptomfree subjects ( 9 women, 58 men; aged $32.0 \pm 12.3$ years; BMI $\left.22.9 \pm 3.2 \mathrm{~kg} / \mathrm{m}^{2}\right)$.

Statistical analysis. An analysis of variance for repeated measures was carried out on the differences between the RRAs before and after therapy readjustment at 10, 20, 30, 40, and $50 \mathrm{~min}$ postprandially. The influences of the pre-prandial blood glucose concentration and that of the increase recorded 15,30 and $45 \mathrm{~min}$ postprandially over pre-prandial values (area under curve) were accounted for by using the differences between the values before and after therapy readjustment as covariables. To analyse the shape of the emptying curves, regression analyses for the differences between the RRAs as recorded before and after therapy readjustment at 10, 20,30, 40, and 50 min postprandially were carried out, which accounted also for the above-mentioned covariables. In addition, correlation analyses between the RRA at 50 min and $\mathrm{HbA}_{1 \mathrm{c}}$, preprandial blood glucose concentration and that at $60 \mathrm{~min}$ after meal ingestion, respectively, as well as two-sided $t$ tests for paired observations were carried out. The zero hypothesis was rejected at an error probability of $p$ less than 0.05 (2tailed).

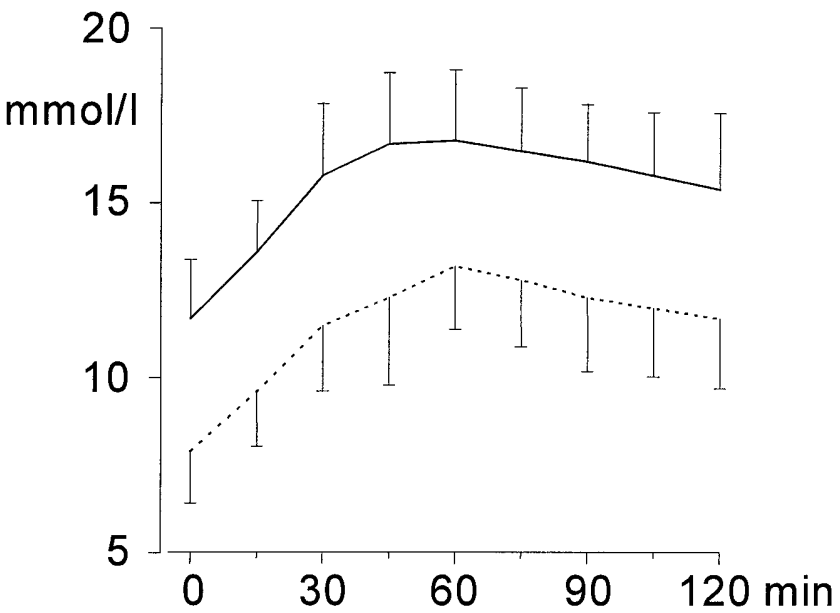

Fig. 1. Blood glucose concentrations before ingestion of the semisolid standard meal $(0 \mathrm{~min})$ and 15 to $120 \mathrm{~min}$ after the end of meal ingestion. Solid line, before therapy readjustment; broken line, after therapy readjustment (means \pm SD)

\section{Results}

Blood glucose concentrations pre-prandially as well as at every postprandial time-point were considerably lower after than before therapy readjustment in all patients. The mean pre-prandial and postprandial glucose concentrations differed significantly $(t$ (9) $=9.12, p<0.0001$; Fig. 1$)$. Despite the significantly lowered blood glucose, gastric emptying was virtually unchanged and the mean emptying curves did not differ (Fig.2). This was confirmed by the regression analyses which showed that none of the differences between the RRAs recorded before and after therapy readjustment at 10 to 50 min postprandially differed significantly from zero. The mean AUCs at $50 \mathrm{~min}$ were $4277 \pm 359$ and $4139 \pm 278$ units, respectively. The mean AUC before therapy readjustment was significantly larger than that of the healthy subjects $(t$ $(75)=2.95, p<0.01)$.

The analysis of variance yielded no hint that the course of emptying over time was systematically changed $[F(4,28)=2.21$, NS $]$. There was no influence of the differences between the pre-prandial glucose concentrations as recorded before and after therapy readjustment on emptying $[F(1,7)=0.31$, NS]. The differences between increases in blood glucose concentrations over those pre-prandial, as recorded before and after readjustment, also had no influence on emptying $[F(1,7)=0.26$, NS $]$. The preprandial blood glucose concentration was not related to the RRA at 50 min postprandial $[r(18)=0.099$, NS]. Further, the absolute blood glucose concentrations attained $60 \mathrm{~min}$ postprandially were not related to the RRAs at $50 \mathrm{~min}[r(18)=0.086$, NS $]$. There was also no relation between the RRA at $50 \mathrm{~min}$ as recorded before therapy readjustment and the $\mathrm{HbA}_{1 \mathrm{c}}$ at that point $[r(8)=0.160, \mathrm{NS}]$. Fasting insu- 


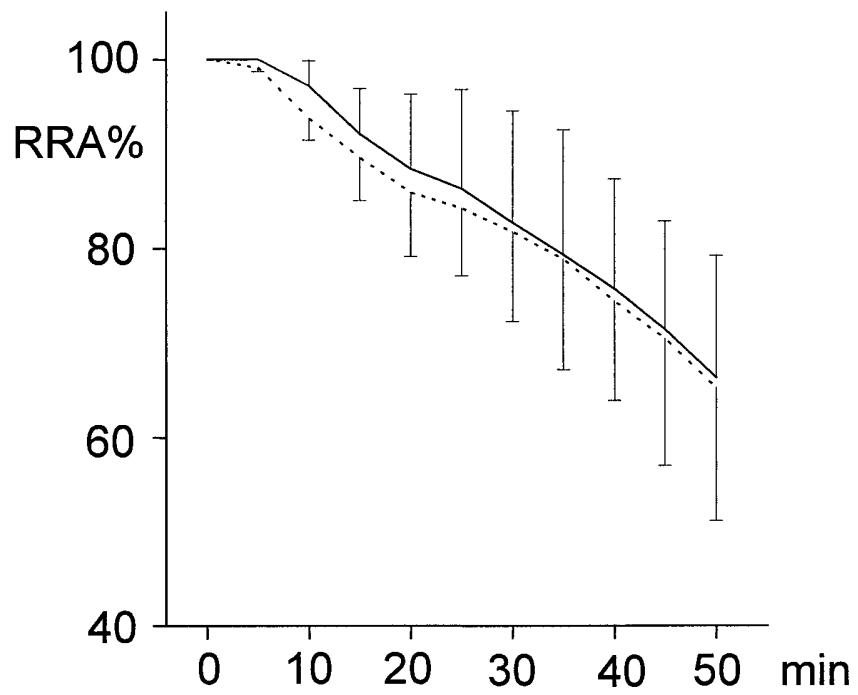

Fig. 2. Gastric emptying of the semisolid standard meal before (solid line) and after (broken line) therapy readjustment (means $\pm \mathrm{SD}$ )

lin concentrations before and after therapy readjustment (means, $8.0 \pm 4.8$ and $7.0 \pm 3.6 \mathrm{mU} / \mathrm{l}$, respectively) did not differ $[t(8)=0.93, \mathrm{NS}]$.

\section{Discussion}

Because gastric emptying remained unaffected by the significantly lower pre-prandial and postprandial blood glucose concentrations, which resulted from therapy readjustment, this does not speak for a major impact of actual, longer-lasting, naturally occurring blood glucose concentrations upon the emptying in patients with Type II diabetes. Our observations contrast with reports of an association between high glucose concentrations and slow emptying in healthy people $[1,2,10]$ as well as in Type I diabetic patients $[4,6]$. Most of the latter studies $[1,2,4]$ investigated, however, the influence of experimentally induced short-term and massive increases in blood glucose concentrations, whereas we evaluated the impact of sustained but in relation modestly heightened concentrations and of their reduction. No relation between fasting blood glucose concentration and gastric emptying was also found in previous studies in patients with Type I diabetes [5, 7, 9]. Against a major importance of pre-prandial blood glucose concentration also speaks the lack of a relation between emptying and $\mathrm{HbA}_{1 \mathrm{c}}$ shown in this and an earlier study [9].

The emptying rates observed also were not influenced noticeably by the extent of the postprandial increase in blood glucose concentration: neither the absolute glucose concentration attained nor the extent of increase above those pre-prandial corresponded to the emptying rate. This finding is not consistent with earlier observations made in subjects who had ingested liquid [6, 7, 10] and semisolid meals [9], which suggested a direct relation between emptying rate and increase in blood glucose concentration. The discrepancy could be explained by most of these investigations having been made, in contrast to the present one, in patients with Type I diabetes $[7,9]$ or in a group consisting predominantly of such patients [6]. The latter study in 86 patients with Type I and Type II diabetes also indicated that there is no close relation between postprandial blood glucose concentration and emptying: although liquid was emptied slower in patients with a mean plasma glucose concentration greater than $15 \mathrm{mmol} / \mathrm{l}$ than in those with a mean concentration less than $15 \mathrm{mmol} / \mathrm{l}$, solid meal components were not emptied differently [6].

In conclusion, the data do not support a major impact of actual, longer-lasting, naturally occurring blood glucose concentrations upon the rate of gastric emptying in patients with Type II diabetes.

\section{References}

1. MacGregor IL, Gueller R, Watts HD, Meyer JH (1976) The effect of acute hyperglycemia on gastric emptying in man. Gastroenterology 70: 190-196

2. Oster-Jørgensen E, Pedersen SA, Larsen ML (1990) The influence of induced hyperglycaemia on gastric emptying rate in healthy humans. Scand $\mathrm{J}$ Clin Lab Invest 50: 831-836

3. Schvarcz E, Palmér M, Åman J, Horowitz M, Stridsberg M, Berne C (1997) Physiological hyperglycemia slows gastric emptying in normal subjects and patients with insulindependent diabetes mellitus. Gastroenterology 113: 60-66

4. Fraser RJ, Horowitz M, Maddox AF, Harding PE, Chatterton BE, Dent J (1990) Hyperglycaemia slows gastric emptying in Type I (insulin-dependent) diabetes mellitus. Diabetologia 33: 675-680

5. Wright RA, Clemente R, Wathen R (1985) Diabetic gastroparesis: an abnormality of gastric emptying of solids. Am J Med Sci 289: 240-242

6. Jones KL, Horowitz M, Wishart JM, Maddox AF, Harding PE, Chatterton BE (1995) Relationships between gastric emptying, intragastric meal distribution and blood glucose concentrations in diabetes mellitus. J Nucl Med 36: 2220-2228

7. Lyrenäs EB, Olsson EHK, Arvidsson UC, Örn TJ, Spjutii JH (1997) Prevalence and determinants of solid and liquid gastric emptying in unstable type I diabetes. Diabetes Care 20: 4-13

8. Merio R, Festa A, Bergmann H et al. (1997) Slow gastric emptying in type I diabetes: relation to autonomic and peripheral neuropathy, blood glucose, and glycemic control. Diabetes Care 20: 419-423

9. Stacher G, Schernthaner G, Francesconi M et al. (1999) Cisapride versus placebo for 8 weeks on glycemic control and gastric emptying in insulin-dependent diabetes: a double blind cross-over trial. J Clin Endocrinol Metab 84: 2357-2362

10. Horowitz M, Edelbroek MAL, Wishart JM, Straathof JW (1993) Relationship between oral glucose tolerance and gastric emptying in normal healthy subjects. Diabetologia 36: 857-862 